\title{
SIMULACIÓN BIDIMENSIONAL NUMÉRICA DEL TRANSPORTE DE SOLUTOS EN LA COSTA DEL ACUÍFERO SANTO DOMINGO, ENSENADA; B .C. MÉXICO
}

\author{
Mariano Norzagaray Campos ${ }^{1}$, Jaime Herrera Barrientos ${ }^{3}$, \\ Fernando Herrera Barrientos ${ }^{3}$, Patricia Muñoz Sevilla ${ }^{1}$, Edgar Yuri Mendoza ${ }^{4}$, \\ Luis Capurro-Filograsso ${ }^{2}$
}

${ }^{1}$ Centro Interdisciplinario de Investigación para el Desarrollo Integral Regional (CIIDIR). Carretera a Las Glorias km 1, Guasave, Sinaloa. c. p 81100, E-Mail : mnorzaga@ipn.mx.

${ }^{2}$ Cinvestav Mérida, E-Mail: lcapurro@mda.cinvestav.mx

${ }^{3}$ Centro de Investigación Científica y de Educación Superior de Ensenada, Km 107 carr. Tijuana-Ensenada. Baja California, México E-Mail: jherrera@ cicese.mx. ${ }^{4}$ Comisión Nacional del Agua, Mexicali B.C. México.

\begin{abstract}
The Santo Domingo coastal aquifer, is a hydrological reserve in the regional urban development of Vicente Guerrero, south of Baja California, facing overexploitation, pollution and saline intrusion problems. This paper aims to evaluate pollution of the underground waters through "Total Dissolved Solids" (TDS) as indicator. Field data cover the period 1977 to January 1997 and show gradual deterioration of water quality, due to attend demands for agriculture, human and industrial purposes. Integration of hydrogeological and hydrogeophysical techniques provided a numerical simulation model which shows that within five years (2008) total TDS will increase considerably in the coastal aquifer.
\end{abstract}

RESUMEN: El acuífero costero de Santo Domingo es una reserva regional en el desarrollo urbano de la región de Vicente Guerrero al sur de Baja California, México. En la actualidad tiene problemas debido a la sobreexplotación de sus aguas, a la contaminación y a la intrusión salina. Para evaluar esta problemática, la presente investigación analiza la contaminación de las aguas subterráneas utilizando como indicador la concentración de sólidos totales disueltos (SDT) del acuífero mencionado. Los datos comprenden el período de 1977 hasta enero 1997 y revelan el deterioro gradual de la calidad del agua, relacionado con bombeos constantes del acuífero para atender la demanda hídrica de la agricultura, consumo humano e industrial. La integración de técnicas hidrogeológicas e hidrogeofísicas permitieron realizar una simulación numérica, lo cual indica que dentro de 5 años, es decir en el año 2008, la concentración de sólidos totales disueltos (SDT) aumentará significantemente en el área de la costa del acuífero de Santo Domingo.

\section{INTRODUCCIÓN}

En este trabajo se modeló la concentración de las aguas subterráneas considerando su evolución espacial y temporal (Pricket \& Lonnquist, 1971). Para ello se empleó el programa de cómputo llamado MOC2SURF (Konikow \& Bredehoeft, 1983). El área de estudio tiene 4 km 
de largo por $3 \mathrm{~km}$ de ancho. Se utilizó esta área porque la dinámica de la intrusión salina es mayor.

El modelaje del transporte de solutos en un acuífero tiene muchas aplicaciones en beneficio de la comunidad que vive en la región, ya que es la base para planear el manejo y conservación de los recursos naturales, sin poner en peligro la salud de sus habitantes.

Para realizar el modelado de transporte de solutos en un medio poroso, es necesario conocer lo siguiente (Custodio \& Llamas, 1983): geometría del acuífero, transmisividad (T), conductividad hidráulica (K), coeficiente de almacenamiento (S) (Villanueva \& Iglesias, 1984), coeficiente de dispersión hidrodinámica (Prickens \& Grisak, 1981), condiciones iniciales y de frontera, tanto para la altura piezométrica como para la concentración. Mediante la técnica del modelado numérico se pretende conocer la dinámica de funcionamiento del sistema acuífero, de tal forma que se simulen escenarios de los cambios espacio temporales de la concentración hasta el año 2008.

\section{LOCALIZACIÓN DEL ÁREA DE ESTUDIO}

El área de estudio se localiza en el municipio de Ensenada, estado de Baja California México y se sitúa a $160 \mathrm{~km}$ al sur de la ciudad de Ensenada. Se comunica mediante la carretera federal No. 1, Tijuana - La Paz. El área estudiada queda comprendida entre las coordenadas $30^{\circ} 41^{\prime}$ '00" y $30^{\circ} 44^{\prime} 42^{\prime}$ ' de latitud norte y $116^{\circ} 03^{\prime} 28^{\prime \prime}$ y $116^{\circ} 01^{\prime} 47^{\prime}$ " de longitud oeste $y$ tiene una superficie de $12 \mathrm{~km}^{2}$ aproximadamente.

El valle de Santo Domingo limita al Norte con el valle de Camalú, al Sur su límite es con el valle de San Quintín, al Este con la sierra de San Pedro Mártir y al oeste con el océano Pacífico (Fig. 1). El área a modelar es una planicie costera cortada por el arroyo Santo Domingo cuya dirección es este-oeste. Tiene una topografía de planicie costera suave con pendiente hacia el mar, lugar que los pobladores denominan "La Bahía San Ramón".

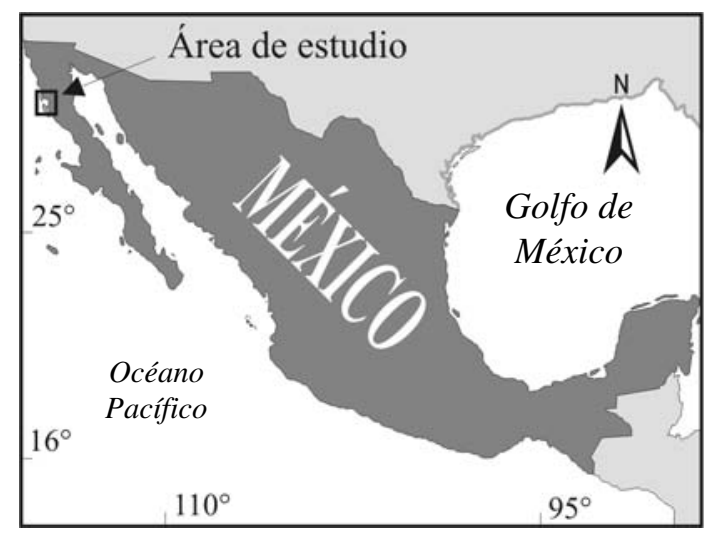

Fig. 1: Localización del área de estudio.

\section{GEOLOGÍA DEL ÁREA DE ESTUDIO}

Raisz (1964) determinó que la zona queda comprendida dentro de la provincia fisiográfica de Baja California. En el valle existen dos prominencias que rigen en gran medida la dinámica del agua subterránea: loma San Ramón y Punta Gorda. Están constituidas predominantemente por conglomerados y areniscas de la Formación Rosario.

Los materiales del acuífero son de tipo aluvial, lacustre y de litoral. Los sedimentos aluviales están constituidos de arenas con granulometría que varía de gruesa a finas, y los lacustres son de tamaño fino incluyendo los limos y las arcillas, y se localizan en las cercanías de la "bahía San Ramón". Como depósitos de litoral se tienen arenas de tamaño fino a medio bien clasificadas. Por otro lado, la presencia del lago de La Bocana Santo Domingo es una fuente de agua dulce en la época de estiaje y, a su vez, constituye un elemento importante en el aporte de agua al acuífero. El basamento geológico del área del valle de Santo Domingo son gneises en la facies de esquistos verdes (los cuales afloran en el límite oriental del valle), así como granodioritas con edad comprendida entre Cretácico y Paleozoico (Soto, 1992).

\section{ESTUDIOS PREVIOS}

La Comisión Nacional del Agua (CNA, 1977) realizó campañas sobre la química (aniones 
y cationes) del agua subterránea en el valle tales como sólidos totales disueltos medidos en ppm (mg/l). Se observa en ellos que las concentraciones variaban de 350 a $600 \mathrm{ppm}$, por lo cual el agua subterránea quedaba clasificada como agua de tipo dulce. En la figura 2, se pueden observar los cambios de los sólidos totales disueltos para 1977. En ese mismo año, la CNA realizó una campaña de prospección geofísica la cual comprendía 23 sondeos eléctricos verticales (SEV's), y para ello se utilizó un arreglo electródico Schlumberger con equipo SCINTREX de 1500 watts de potencia.

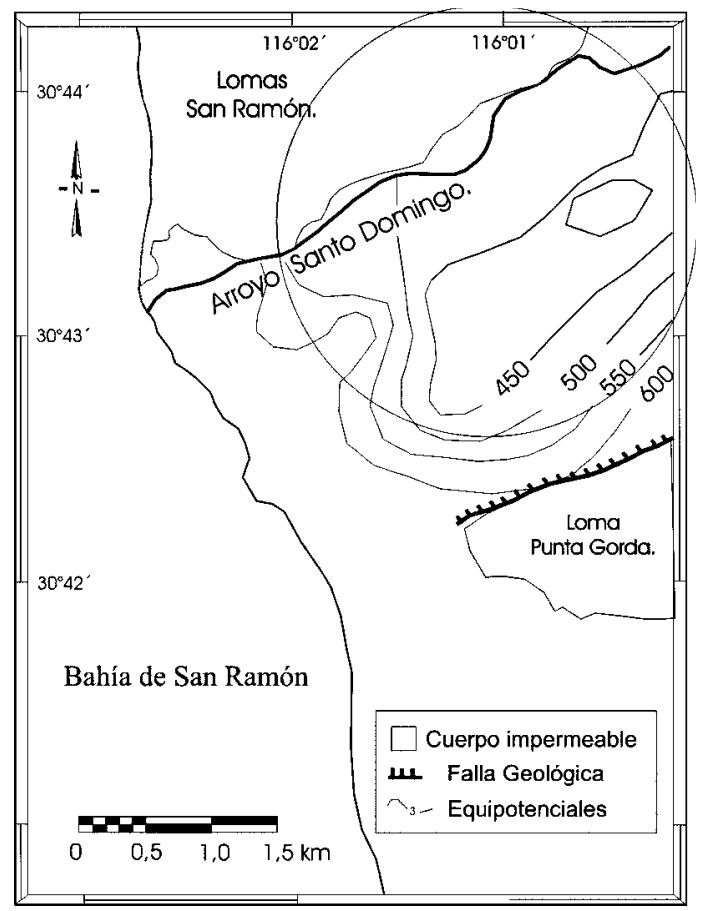

Fig. 2: Concentración observada en sólidos totales disueltos para 1977, en mg/l. El círculo indica la zona de monitoreo realizada por la Comisión Nacional de Agua (CNA) para los sólidos totales disueltos, en al año 1977.

Los sondeos mencionados, fueron reinterpretados de manera cuantitativa, y se determinó la geometría del acuífero (Herrera, et al., 1995). De igual forma, Herrera et al. (1995) determinaron la geometría del acuífero libre de Santo Domingo a través de la correlación entre la técnica de sondeos eléctricos verticales y la técnica de inducción electromagnética (EM-34). Otro estudio sobre la geohidrología del valle lo realizó
Gutiérrez (1992); en éste se describe la geología de la zona, la elaboración de pruebas de bombeo, determinación del coeficiente de almacenamiento, así como las direcciones principales de flujo del agua subterránea.

Mendoza (1996) realizó el modelado de flujo en el valle de Santo Domingo conforme a los principios establecidos por Bear (1972) y utilizó un esquema numérico en diferencias finitas (Pricket \& Lonnquist, 1971). Herrera et al. (1997) estimaron la porosidad y la geometría en la porción próxima al litoral del acuífero. Respecto a la porosidad, para su determinación se empleó la ley de Archie. Para satisfacer la ecuación planteada en la ley de Archie, se utilizó la resistividad de la formación obtenida por el método electromagnético EM-34 y la resistividad del agua de la formación acuífera a través de medidas de conductividad eléctrica de muestras de agua subterránea.

\section{METODOLOGÍA}

Para la realización del modelado de transporte de solutos se seleccionó el software MOC2SURF, realizado por Konikow \& Bredehoeft (1978) y actualizado en la versión 1995. Este programa se ha utilizado ampliamente en la literatura (Konikow \& Bredehoeft, 1984; Konikow, 1977 y Calvache \& Pulido, 1991).

En la simulación numérica del valle de Santo Domingo se consideran los fenómenos de advección y difusión (Robinson \& Stokes, 1965) existentes en la expresión general de transporte de solutos (Reddell \& Sunada, 1970). En el caso particular del coeficiente de dispersión hidrodinámica (D), se utilizaron valores próximos a los publicados en la literatura $\left(0,018\right.$ y $\left.0,055 \mathrm{~m}^{2} / \mathrm{s}\right)$, para acuíferos aluviales (Prickens \& Grisak, 1981).

Para observar la sensibilidad de los cambios de la concentración de los sólidos totales disueltos con base en la variación del coeficiente de dispersión hidrodinámica se hicieron pruebas durante la simulación numérica utilizando el rango arriba mencionado, y se encontró que la concentración es prácticamente insensible a los cambios de la dispersividad hidrodinámica (a). 
La ecuación de transporte utilizada para la simulación numérica del acuífero de Santo Domingo, reportada varias veces en la literatura (Reddell \& Sunada, 1970; Bear, 1972; Bredehoeft \& Pinder, 1973; Konikow \& Grove, 1977) en forma bidimensional, es la siguiente:

$$
\frac{\delta C}{\delta t} b=\frac{\delta}{\delta x_{i}}\left[b D_{i j} \frac{\delta C}{\delta x_{j}}\right]-\frac{\delta}{\delta x_{i}}\left(b C v_{i}\right)-\frac{C^{\prime} W}{\varepsilon}, i j=1,2
$$

Donde:

$C=$ Concentración del soluto, $\mathrm{ML}^{-3}$

$D_{i j}=$ Tensor del coeficiente de dispersión hidrodinámica, $\mathrm{L}^{2} \mathrm{~T}^{-1}$

$b=$ Espesor de la zona saturada del acuífero, $\mathrm{L}$

$C^{\prime}=$ Concentración de la sustancia inyectada y/o extraída del acuífero, $\mathrm{ML}^{-3}$

$W=$ Carga o descarga positivo para la extracción y negativo para la inyección, $\mathrm{LT}^{-1}$

$V_{i}=$ Es la velocidad lineal del flujo, $\mathrm{LT}^{-1}$

En la ecuación anterior los índices $i, j$ varían de 1 a 2, ya que se está asumiendo en la simulación numérica la bidimensionalidad de los cambios de la concentración.

Para resolver esta ecuación con valores de la frontera, se utilizó el método de diferencias finitas en mallas regulares, aplicando direcciones alternadas de gran utilidad para regiones de geometría irregular. De esta forma, se obtuvo el campo de velocidades el cual se utiliza en la ecuación de transporte, la cual se resolverá con el método de las características (Garder \& Peaceman, 1964; Abbott, 1966; Scott, 1987). Dentro de esta ecuación general de transporte se toman en cuenta la presencia de fuentes contaminantes por efectos de inyección o vertido de residuos, así como también la acción de bombeos (Heath, 1991).

La idea fundamental de este modelado numérico es la representación real del sistema acuífero de Santo Domingo en forma aproximada. Lo cual resulta de la comparación del comportamiento real de la concentración con la calculada a través del modelo numérico. Para usar el paquete de cómputo MOC2SURF se analizaron las condiciones establecidas en la literatura (Konikow \& Bredehoef, 1984; Bredehoeft \& Pinder, 1973), comparándolas con las existentes en el acuífero, y éstas son las siguientes:
1) La ley de Darcy se cumple sin ningún problema, ya que el acuífero estudiado es granular, situación válida en esta ley; además, los cambios en la piezometría son los que originan el flujo (gradiente hidráulico).

2) La conductividad hidráulica $(k)$ y la porosidad $(f)$ son constantes en el tiempo. Esta última se considera uniforme en el espacio. Los cambios temporales de estos dos parámetros hidrológicos se pueden considerar pequeños en este acuífero costero, ya que no existen fenómenos geológicos que originen una porosidad secundaria ni alteraciones importantes en la conductividad hidráulica, a la escala de tiempo que se trabaja (años).

3) Se considera que los gradientes de la viscosidad, densidad y temperatura no afectan el campo de velocidades en el acuífero.

4) No existen reacciones químicas que afecten la concentración del soluto; las propiedades del fluido ni las del acuífero. Hasta el momento, en la literatura, no se reportan cambios importantes asociados a posibles reacciones químicas de los sólidos totales disueltos en este valle.

5) Los efectos por difusión iónica y molecular tienen una contribución despreciable a la dispersión total del flujo. En éste acuífero el transporte de solutos debido a la difusión iónica y molecular es menos significativo que el transporte convectivo y por dispersión (Fetter, 1992), por lo que esta suposición se apega en buena forma al problema aquí estudiado.

6) Las variaciones en la vertical de la concentración y la piezometría son despreciables, por lo que se tiene un flujo y transporte bidimensional con componentes horizontales. Para éste acuífero las dimensiones horizontales, con respecto a las verticales, son mucho más grandes, de tal forma que el flujo se puede considerar horizontal (Calvache \& Pulido, 1991) y, como la principal componente de transporte es la convectiva, entonces el suponer que la concentración también es horizontal resulta un planteamiento aceptable. 
El acuífero es homogéneo e isotrópico con respecto a la dispersividad longitudinal y transversal. La literatura reporta, que en otros valles las pruebas del coeficiente de dispersividad calculado en el laboratorio presentan rangos de variación de uno a dos órdenes decimales a lo sumo. Es por ello que la condición de homogeneidad e isotropía es una suposición apropiada para este trabajo. Además, como el efecto difusivo es pequeño se ha despreciado, por lo que a la ecuación de transporte se le llama ecuación de dispersión advección.

Para utilizar el programa de cómputo MOC2SURF es necesario contar con la información en forma discreta; para ello se discretiza el área en celdas de $500 \mathrm{~m} \times 500 \mathrm{~m}$, resultando una malla de 9 x 12 nodos. El tamaño se ha diseñado tomando en cuenta que los cambios geológicos existentes en el área son pocos, esto conforme a los resultados geofísicos. Por otra parte, la separación entre los pozos de monitoreo es aproximadamente esa distancia.

Las condiciones de frontera impuestas al programa son las siguientes:

- Límite impermeable en dirección perpendicular a las Lomas San Ramón y Punta Gorda. Esto debido a los conglomerados de la Formación Rosario, por lo que los cambios de la concentración en dirección a dichas fronteras geológicas es nulo.

- Condición a potencial y concentración constante en el mar.

- Gradiente de concentración y piezométrico en el límite Este del área de estudio.

Esta consideración se usa, ya que no existe una frontera natural (geológica). Como condición inicial para el flujo y transporte de masa se consideró la serie piezométrica (Fig. 3) y de concentración de sólidos totales disueltos de agosto de 1996 (Fig. 4). Para la calibración del modelo de transporte se usaron las series de concentración de septiembre de 1996 (Fig. 5) y enero de 1997 (Fig. 7).

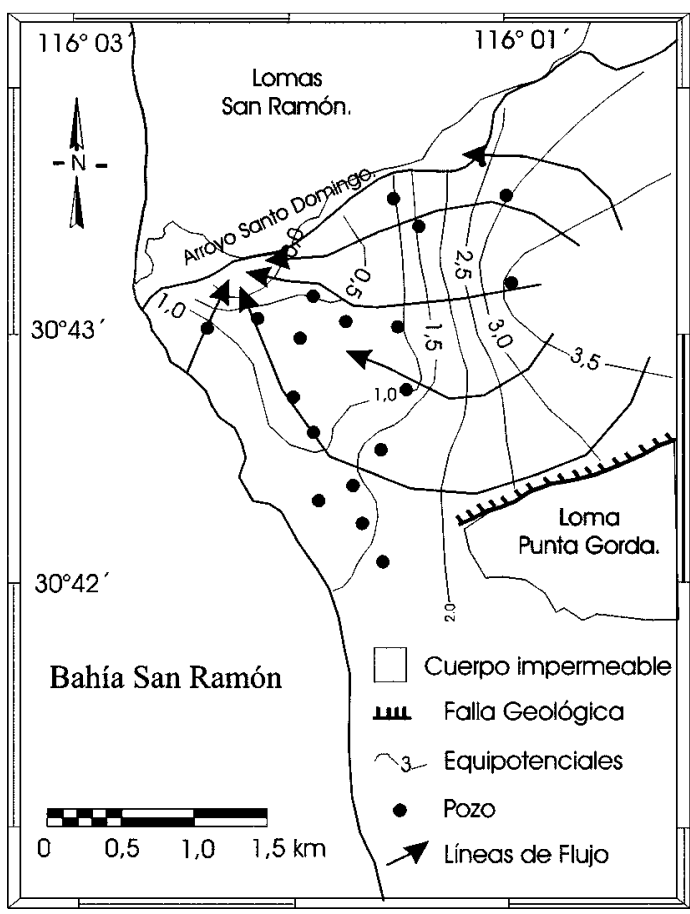

Fig. 3: Piezometría observada correspondiente a agosto de 1996, isocontornos en metros.

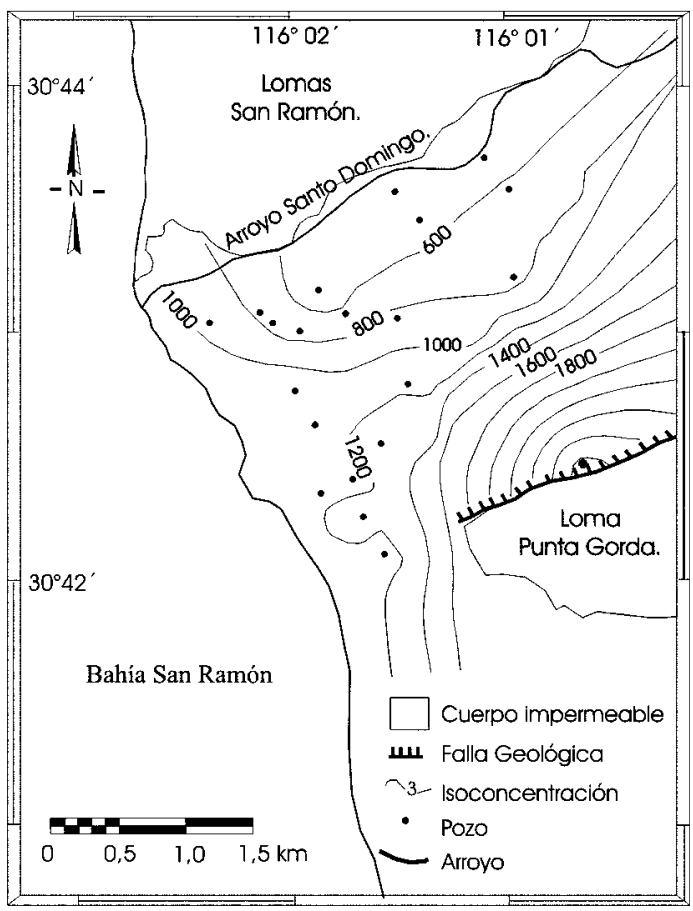

Fig. 4: Concentración observada en sólidos totales disueltos para agosto de 1996, en mg/l. 


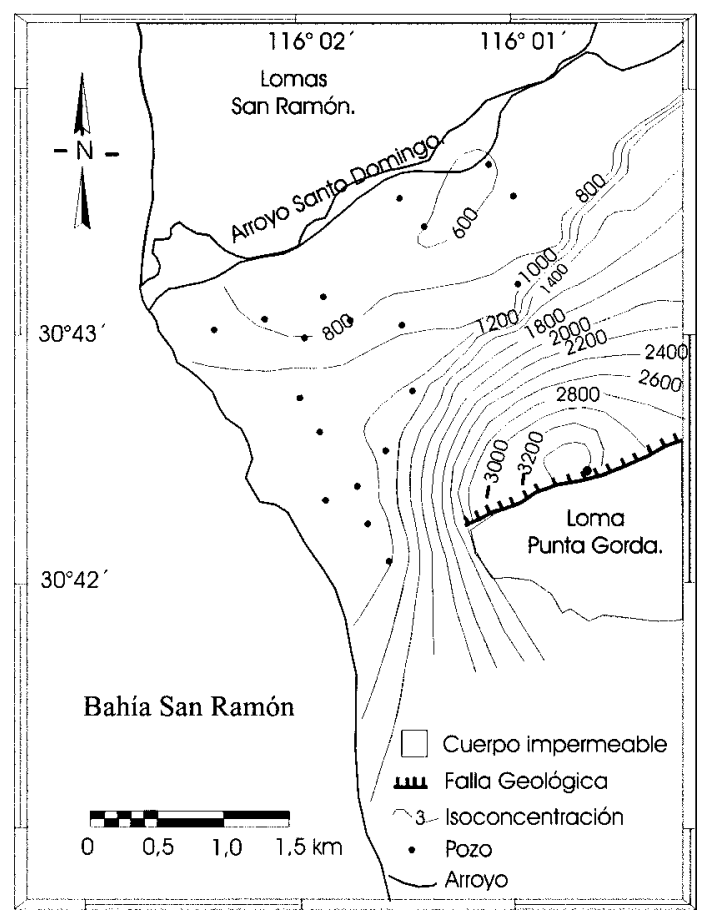

Fig. 5: Concentración observada en sólidos totales disueltos para septiembre de 1996 en mg/l.

Para generar las series piezométricas se realizó la nivelación topográfica de 32 obras mediante un teodolito electrónico DT5 acoplado a un distaciómetro RED2L de la casa Sokkia. Para la nivelación se utilizó el banco de nivel no. 975 del Instituto Nacional de Estadística Geografía e Informática (INEGI), ubicado a un lado de la puerta de la iglesia del poblado Colonia Vicente Guerrero. Los datos del banco de nivel son latitud $30^{\circ} 43^{\prime} 05^{\prime}$, longitud $115^{\circ} 59^{\prime} 28^{\prime \prime}$ y altura de 26,186 m s.n.m. Una vez niveladas las obras y medida la profundidad del nivel estático para los meses de agosto y septiembre de 1996, así como enero de 1997, se obtiene la altura piezométrica al restar de la altura de brocal la profundidad del nivel del agua para cada período.

En la figura 3 se observa como el flujo principal tiene una dirección NW; asimismo, la presencia de una componente de flujo de dirección NW asociada a la laguna Figueroa, la cual es intermitente y se ha recargado durante los años de mayor precipitación como 1993, manteniendo un tirante de agua superficial de hasta 4

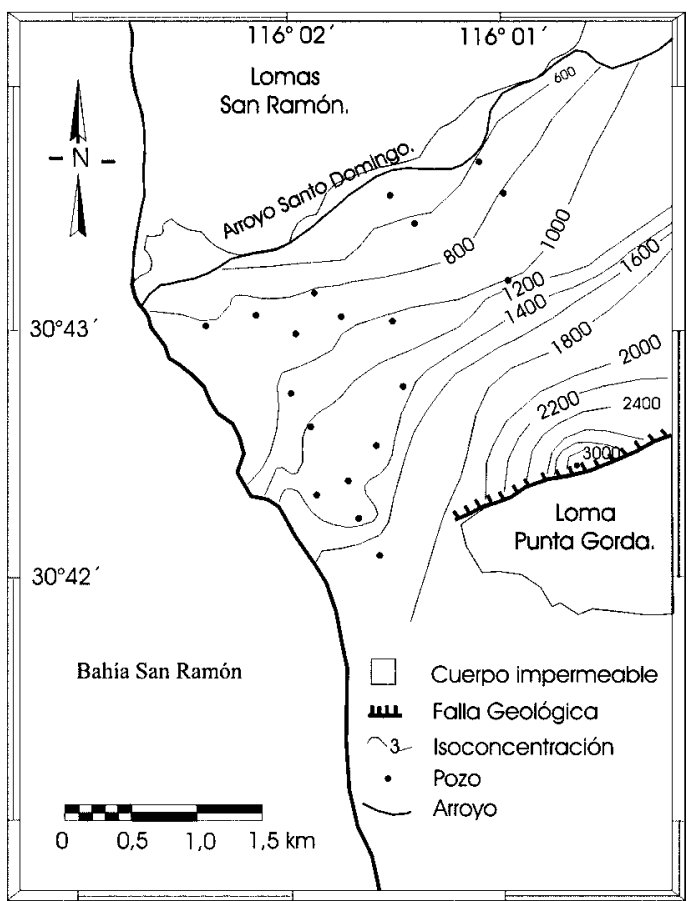

Fig. 6: Concentración observada en sólidos totales disueltos para enero de 1997 en mg/l.

metros. Dicha laguna se ubica al SW de loma San Ramón. En la figura 4 se muestra que el agua de mejor calidad está en las proximidades al arroyo Santo Domingo, empeorando la calidad hacía el interior del acuífero y en las proximidades a loma Punta Gorda; nótese, como después de 19 años la concentración de sólidos totales ha cambiado significativamente, esto debido a las extracciones asociadas a la actividad agrícola. La serie de septiembre de 1996 (Fig. 5) muestra la misma conducta que la de agosto de 1996. Para enero de 1997, la concentración de STD ha aumentado en 200 ppm (Fig. 6), tanto en las proximidades al arroyo como a loma Punta Gorda. La observación de los antecedentes de la zona ha permitido establecer el cambio en concentración suscitado en sus aguas subterráneas y, a su vez, detectar el problema de intrusión salina en el área debido a sobre bombeo.

Otro de los parámetros a considerar dentro de la simulación numérica son los bombeos ejercidos en la zona de estudio, pero hasta el momento no se tiene un control temporal sobre el caudal 
de extracción del recurso hídrico de los pozos en el valle debido a la ausencia de medidores de gasto en los pozos. Por lo que el caudal de extracción se obtuvo mediante prácticas expeditivas, es decir, con métodos que permiten estimar el caudal que circula por una tubería sin necesidad de instalación de aparatos de medida, como la práctica propuesta por Bennison (Villanueva \& Iglesias, 1984); para ello se midió la distancia horizontal entre la extremidad del tubo donde brota el agua y un punto situado exactamente a un pie por encima de la caída de agua y la sección de la tubería, obteniéndose así el gasto con la tubería llena. Si la tubería de extracción no estaba llena, el resultado de la medición se multiplicó por el cociente entre el espesor del cuerpo de agua de la tubería y el diámetro de ésta. Las mediciones se hicieron cinco veces en cada pozo y se obtuvo el valor promedio, el cual se empleó en el cálculo del caudal. Se consideró una equivalencia de funcionamiento de 12 horas por día en cada pozo. Este caudal se considera constante durante todo el proceso de simulación

\section{RESULTADOS}

El modelo se calibró con las series de concentración de sólidos totales disueltos de septiembre de 1996 (Fig. 5) y enero de 1997 (Fig. 7). Para ello se consideraron como condiciones iniciales las series de agosto de 1996, tanto para piezometría (Fig. 3) como concentración de STD (Fig. 4). Las figuras 7 y 8 muestran las concentraciones calculadas por el modelo para los meses de septiembre de 1996 y enero de 1997. Ambas reproducen las tendencias y valores de las series observadas para las mismas fechas y mostradas en las figuras 5 y 6 . Además del criterio cualitativo visual de calibración, se consideró el cuantitativo tomando en cuenta los criterios de calibración indicados por Anderson \& Woessner (1991), a saber, error medio (ME), error medio absoluto (MAE) y error raíz cuadrático medio (RMS). En el cuadro 1 se muestran los cocientes de los errores indicados con respecto a la concentración máxima observada para las series de septiembre de 1996 y enero de 1997, así como

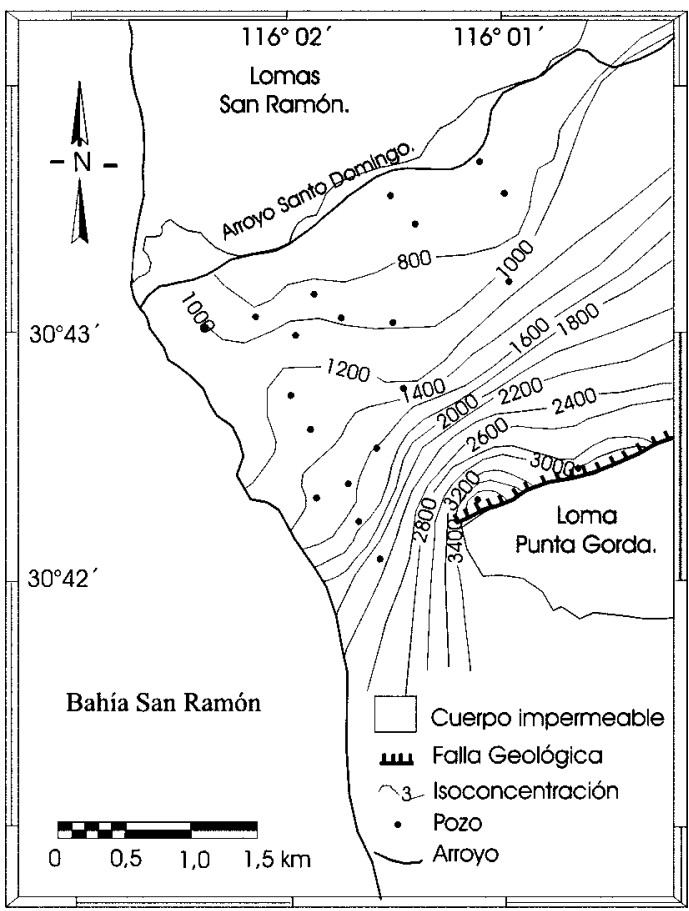

Fig. 7: Concentración calculada por el modelo correspondiente a septiembre de 1996 en $\mathrm{mg} / \mathrm{l}$.

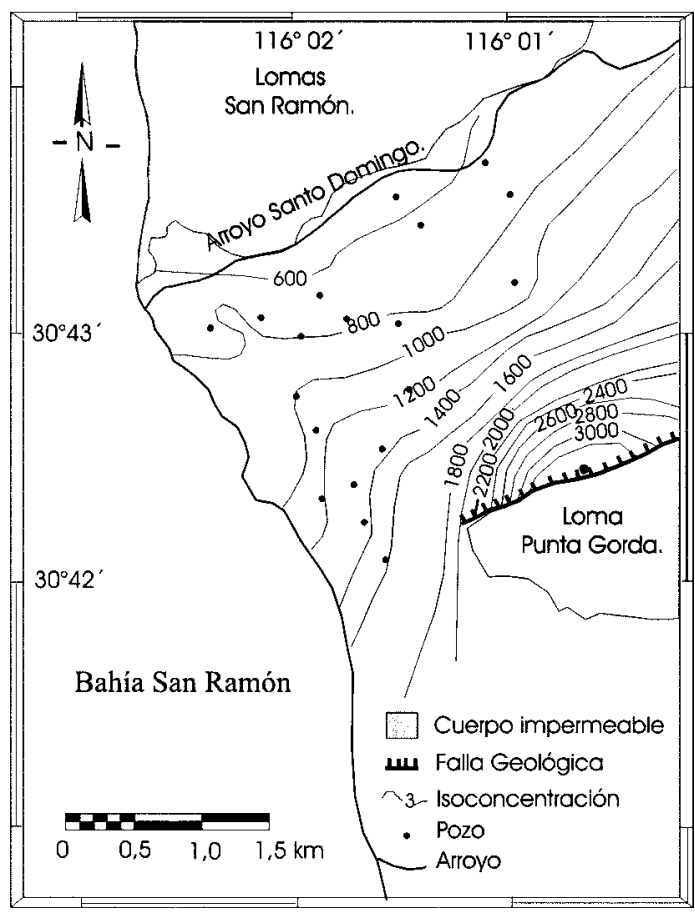

Fig. 8: Concentración calculada por el modelo correspondiente a enero de 1997 en mg/l. 
los errores promedios correspondientes. El ME representa el error menor, mientras que el RMS el mayor; esto debido al efecto de las fronteras, especialmente en loma Punta Gorda. La magnitud de los errores de calibración permite considerar como apropiado el proceso de calibración, por lo que la figura 9 muestra la predicción de los sólidos totales disueltos para el año 2008, observándose como el avance de la intrusión salina es de tal forma, que la aportación del agua del arroyo no logra mejorar la calidad del agua; esto en gran parte debido a que el flujo subterráneo se reduce debido a las extracciones en las porciones NE del arroyo, así como a las extracciones en las proximidades a la línea de costa. Por concentraciones mayores a $1000 \mathrm{mg} / \mathrm{l}$, la calidad del agua es ligeramente salina (Heath, 1991).

Cabe recordar, que esta predicción se ha hecho considerando el régimen de explotación constante sin tomar en cuenta recargas ni naturales, ni artificiales. Estos resultados señalan

Cuadro 1

Cocientes y valores promedio de los errores para la concentración máxima

\begin{tabular}{cc}
\hline Septiembre de 1996 & Enero de 1997 \\
\hline ME & 0,091 \\
MAE & 0,178 \\
RMS & 0,23 \\
ME & 0,057 \\
MAE & 0,0057 \\
RMS & 0,22 \\
\hline
\end{tabular}

Error promedio: 0,166

Error promedio: 0,094

la sensibilidad del acuífero a la explotación. Los resultados de este modelo sirven para hacer una planeación a corto, mediano y largo plazo, con perspectivas a la recuperación de suelos a través de un uso sostenible del recurso hídrico en la región de Vicente Guerrero. Con perspectivas a la recuperación de la calidad del acuífero, para lograr así su garantía como una reserva regional a futuro, aspecto importante debido a que este sistema hídrico es el único portador de agua dulce para las actividades en general de la zona.

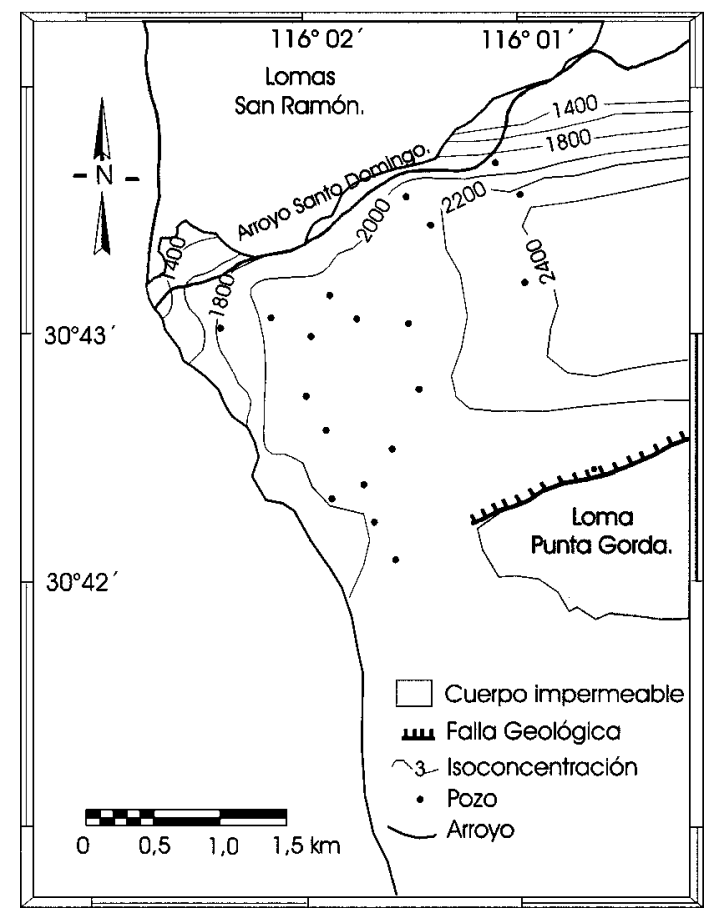

Fig. 9: Predicción de la concentración por el modelo para el año 2008, en mg/l.

\section{CONCLUSIONES Y RECOMENDACIONES}

La presencia de la Bocana Santo Domingo en la época de estiaje constituye un elemento importante en el aporte de agua al acuífero.

Los bombeos persistentes en el área inducen al avance de la intrusión salina hacia el continente. Las porciones más salinizadas no están en contacto directo con el mar. Las más próximas a éste son las menos salinizadas debido al aporte directo del arroyo Santo Domingo.

El acuífero es muy sensible a la contaminación, ya que en cinco meses de observación se mostraron avances de $145 \mathrm{~m}$ de la isocurva 800 $\mathrm{mg} / \mathrm{l}$, así como depresiones del orden de 1,3 m.

Las porciones mas contaminadas del acuífero son las más cercanas a loma Punta Gorda. Remediar éstas por acción natural resulta difícil en tanto no cesen los bombeos en la proximidades al arroyo Santo Domingo.

Se recomienda estudiar la variación temporal de los bombeos, para que en el modelado 
se tomen en cuenta (extracciones), ya que a la fecha se han considerado constantes durante todo el tiempo de modelado. De igual forma, se hace la recomendación para las recargas al acuífero.

Realizar medidas puntuales en el área de la conductividad hidráulica, ya que las calculadas se han basado en gran medida en la extrapolación de la transmisividad hidráulica en áreas cercanas a la de estudio.

Queda abierta la línea de investigación tendiente a determinar el valor del coeficiente de dispersión hidrodinámica y estudio del flujo mediante piezómetros de observación. La importancia de éste revelará el comportamiento de la zona de mezcla.

La base de datos proporcionada permite seguir trabajando sobre el esquema de transporte de masa de otros contaminantes. Por la vocación del área se tendrán los nitratos, pesticidas, fungicidas, herbicidas, entre otros.

Las campañas piezométricas y de contaminantes deben de continuar para un mejor conocimiento, manejo y conservación del acuífero, especialmente los aspectos relacionados con la capacidad de recarga y mecanismos de transporte en el acuífero.

\section{REFERENCIAS}

ABBOTT, M.B., 1966: The method of characteristics. - 239 págs. American Elsevier, New York.

ANDERSON, M.P. \& WOESSNER, W., 1991: Applied groundwater modeling. Simulation of flow and advective transport. - 381 págs. Academic Press, London.

BEAR, J. 1972: Dymanics of fluids in porous media. - 764 págs. American Elsevier, New York.

BREDEHOEFT, J. D. \& Pinder G.F., 1973: Mass transport in flowing groundwater. -Water Resources Res, 9,1: 194-210.

CALVACHE, M.L. \& PULIDO B.A., 1991: Saltwater intrusion into a small coastal aquifer (Río Verde, Almuñecar, southern Spain). - J. Hidrol. 129: 195 -213.

CNA, 1977: Informe técnico del estudio hidrológico de Baja California. - 200 págs. Secr. Agric. Recursos Hidráulicos (ahora CNA), Dep. Aguas Subterráneas, Mexicali, Baja California, México [Inf. Interno].
CUSTODIO, E., \& LLAMAS M. R., 1983: Hidrología subterránea - Tomo I. 1157 págs. Omega, Madrid.

FETTER, C.W., 1992: Contaminant hydrogeology. - 458 págs. Macmillan, New Jersey.

GARDER, A. O. \& PEACEMAN D. W., 1964: Numerical calculation of multidimensional miscible displacement by the method of charateristics. - Soc. Petroleum Eng. J. 4,1: 26-36.

GUTIÉRREZ, S. R., 1992: Estudio geohidrológico del Valle Vicente Guerrero, Baja California Norte. - 86 págs. Univ. Nac. Autónoma de México. [Tesis Lic].

HEATH, R.C., 1991: Basic ground-water hydrology. - 81 págs. Water-supply Paper 2220, Washington..

HERRERA, B. J., HERRERA, B.F \& NORZAGARAY, C. M., 1995: Piezometría y sólidos totales disueltos para el acuífero costero Santo Domingo, Municipio de Ensenada, Baja California, México. Diversas implicaciones. - 85 págs. Centro Invest. Sup. Ensenada, Baja California, México.

HERRERA, B. J., MENDOZA, E.Y. \& HERRERA, B.F., 1995: Determinación de la geometría del acuífero costero Vicente Guerrero, B. C. mediante prospección geoeléctrica. - 41 págs. Centro Invest. Cient. y Educ. Sup. Ensenada, Baja California, México [Inf. técnico].

HERRERA, B.J., NORZAGARAY, C. M, \& HERRERA, B.F., 1997: Estimación de la porosidad en la planicie costera del acuífero Santo Domingo, Municipio de Ensenada Baja California, México. - 68 págs. Centro Invest. Cient. y Educ. Sup. Ensenada, Baja California, México [Inf. técnico].

KONIKOW, L. F. \& BREDEHOEFT, J.D., 1983: Computer model of two dimensional solute transport and dispersion in ground water. - 90 págs. Invest. USGS. United States Gov. Printing Of. Virginia, EEUU.

KONIKOW, L. F. \& BREDEHOEFT, J.D., 1984: Techniques of water-resources Investigations of the United States Geological Survey. - 90 págs. United States Gov. Printing Of. Virginia, EEUU.

KONIKOW, L. F. \& GROVE, D. B., 1977: Derivation of equations describing solute transport in ground water. - 30 págs. USGS water-resources Invest. Virginia, EEUU.

KONIKOW, L. F., 1977: Modeling chloride movement in the alluvial aquifer at the Rocky Mountain Arsenal. Colorado. - 43 págs. USGS Water-Supply. 2044, Reston, Virginia. 
MENDOZA, E.Y., 1996: Simulación numérica del acuífero Vicente Guerrero, B.C., México. - 108 págs. Centro Invest. Cient. y Educ. Sup. Ensenada, Baja California, México [Tesis MSc.].

PRICKENS, J.F. \& GRISAK, G. E., 1981: Scale-dependent dispersion in a stratified granular aquifer. - Water Resources Res. 17, 4: 1191-1211.

PRICKET, T. A. \& LONNQUIST, G.G., 1971: Selected digital computer techniques for grundwater resource evolution. - 62 págs. Bull. Illinois State Water Surv. Illinois.

PINDER, G.F. \& COOPER, H.H., 1970: A numerical technique for calculating the transient position of the saltwater front. - Water Resources Res. 4,5: 1069-1093.

RAISZ, E., 1964: Landforms of Mexico - [2a ed.] 79 págs. Omega, London.

ROBINSON, R.A. \& STOKES, R.H., 1965: Electrolyte solutions. - 67 págs. Butterworth, London.
REDDELL, D. L., \& SUNADA, D.K., 1970: Numerical simulation of dispersion in groundwater aquifers. - 79 págs. Hydrol. Pap. 41. Colorado State Univ. Fort Collins, Colorado.

SCOTT, K.A., 1987: The method of characteristics and solutions of the nonlinear hyperbolic wave equations of sallow water theory. - 39 págs. Univ. California, Santa Cruz.

SOTO, G.R., 1992: Estudio geohidrológico del Valle Vicente Guerrero, Baja California Norte. - 86 págs. Univ. Nac. Autónoma México [Tesis Lic.].

S.P.P- U.N.A.M., 1984: Geología de la República Mexicana. - [2a. ed.] 152 págs. Inst. Nac. Estadística Geogr. Informática (INEGI), México D.F.

VILLANUEVA, M.M. \& IGLESIAS, L.A., 1984: Pozos y acuíferos técnicas de evaluación mediante ensayos de bombeo. - 426 págs. IBERGESA, Madrid. 